\section{Introducing Healthcare in Low-resource Settings}

\section{Chandrakant Lahariya \\ Public Health Specialist, New Delhi, India}

Healthcare resources are often referred to as the means available in a health system to deliver services to the population. Healthcare or health resources, like any other system, can be grouped into three broad categories of infrastructure, material or supplies or consumable, and human resources. Health outcome or status of a population is interplay of all 3 categories of resources, working in tandem to deliver services. There are population-based global norms for resources in a functioning health system. A health system which does not meet the accepted norms can be called as low-resource setting (LRS) for healthcare.

The resources for health are allocated by national governments and the economic condition of the country is a major determinant in health budget allocation. Political instability, public unrest, war, conflicts and natural calamities, all play a major role in the determining the resources for health system. As a rule of thumb, low and middle income countries (LMICs) have at least some form of imbalance in either health infrastructure or supply or in human resources. Thus, LMICs not always but generally equates with lowresource healthcare settings.

The historically low resource allocation for healthcare in LMICs leads to increased disease burden and health service requirement, worsening the situation. Healthcare in LRSs is different from other countries in many aspects: lower investments by the governments, poor infrastructure, limited equipments and medicines, scarce human resources with high turn-over, limited service provision, and large out-of-pocket expenditure. The situation becomes even more complex when discussion moves from simple inputs (infrastructure, materials and human resources) to distribution of health services, fragmentation of services (rural-urban, preventive-curative, major city-small town, motherchild, young-old) in-equities and also on service provision for various health issues, etc.

Low resource settings are the reality and have the highest all-cause mortality, including child and maternal mortalities, thus hampering the overall achievement of Millennium Development Goals. The quantity and quality of healthcare services in LRS is not supposed to be different than any other situation; however, till the resources are increased, the available resources should be effectively and efficiently utilized. The solutions of the local challenges need to be found by research, which is often not conducted due to the low resources/funding. The emic health data is needed rather than the widely available etic data. Though, both of these approaches could be complementary. The view often presented by external observers (in etic) is often colored by the experience in their own settings and has less acceptability by the country program managers. There is emerging need for emic data from LRSs to bring transformational health changes. The sufficient etic data exist and the time for emic to supplement this information is ripe.

Low-resource settings need additional attention as there are higher expectations from these to perform and deliver. The cultural context needs to be understood and addressed and the political leadership in these settings need to be brought on the board. People often get overwhelmed by the absolute number of challenges and the efforts become non-starter because of failure to identify a point to start with. People in LRSs need as good services as in any other settings and they cannot wait indefinitely. The challenges should be addressed immediately and can probably be solved by recognizing healthcare in low-resource settings (HLS) as a separate field of work: that is what this Journal aims at.

I have written in the past that scientific journals have a bigger role to play in healthcare delivery. A reputed and well-focused journal can draw a lot of attention on the part of all stakeholders to bring right changes and become a societal catalyst. ${ }^{1}$ This trend has become increasingly common where journals publish research theme issues, start discussion and debates among stakeholders and changes happen. The lack of availability of local evidence is often cited as a major hurdle in improving health status and introducing new interventions in LRSs. ${ }^{2}$ The gap in knowledge translation and in policy to implementation has been reported often. The 10/90 gap where there is $10 \%$ investment on the problems of $90 \%$ - is also often highlighted. ${ }^{3}$

The limited in-country research capacity, scarce funding and poor quality data results that very little evidence (for action) is generated from LRS. The research work conducted in these settings fails to find a place in reputed journals due to lack of methodological rigour and poor quality data. This has a domino effect and when it comes to decision making, the sufficient local pieces of evidence are not available.

The limited resources make it necessary to optimally utilize the available resources. At a program manager level, this could be achieved by improving focus on efficiency and effectiveness; designing service provision as per the local epidemiology and needs; ensuring equitable distribution; and making
Correspondence: Chandrakant Lahariya, B7/24/2,

First Floor, Safdarjung Enclave Main, Safda-rjung Enclave Main, New Delhi-110029, India.

Tel. +91.98101.60665.

E-mail: c.lahariya@gmail.com

Conflict of interests: the author declares no potential conflict of interests.

Received for publication: 2 January 2013.

Accepted for publication: 13 January 2013.

This work is licensed under a Creative Commons Attribution 3.0 License (by-nc 3.0).

(C) Copyright C. Lahariya., 2013

Licensee PAGEPress, Italy

Healthcare in Low-resource Settings 2013; 1:e1 doi:10.4081/hls.2013.e1

health system responsive to the need of people. Additionally, the cost analyses (cost effectiveness, cost benefit and accounting, etc.) are other sub-optimally utilized tools in these settings.

The publication of Healthcare in Lowresource Settings would provide a platform to researchers and policy makers alike. The Journal aims to publish editorials, commentaries, policy analysis, review articles, original research work and would often have discussions and debates on important health issues with focus on HLS. Opinion articles and viewpoints on topical issues and both qualitative and quantitative research would find place in the Journal. Free access to the research published in HLS is likely to benefit the cause of researchers in LRSs. Healthcare in Lowresource Settings would publish series to build capacity of researchers in LRS in research methodology, cost analyses and epidemiological principles. Healthcare in Low-resource Settings is an idea converted into a Journal to provide a common platform for information sharing and dissemination. It will welcome authors to document best practices and compare health situations in low resources with those in other settings. It is expected that with the time, the pieces of evidence published in this Journal assist in cross-learning to find solutions to common health challenges.

I sincerely hope that a few years down the line, the Journal would have accumulated some path-breaking research evidences to guide the health system in such settings, when it becomes a common platform used by researchers in all countries. The growth and improvement in healthcare will benefit from research evidences in HLS. This is my sincere thought and wish, and all of you are welcome to join this exciting journey.

I really feel privileged to introduce this 
Journal to you. The Journal aims to bring path-breaking changes in health service delivery and healthcare provision in LRSs. It requires your regular contributions and inputs. The journey would be satisfying only if it could improve the health of people in the settings where they need it the most.

\section{References}

1. Lahariya C. Journals have a bigger role to play in the healthcare delivery. Indian $\mathrm{J}$ Community Med 2006;31:120.
2. Sidiqui K, Newell JN. Putting evidence into practices in low-resource settings. B World Health Organ 2005;83:882.

3. Currat LJ, de Francisco A, Nchinda TC. The $10 / 90$ report on health research 2000 . Geneva: Global Forum for Health Research Publ.;2000. 